\title{
LAS DIFERENCIAS ENTRE UNA SOCIEDAD DE PENSAMIENTO NETAMENTE MÁGICO Y OTRA DE PENSAMIENTO RACIONAL
}

\author{
Helena Hernández Martínez \\ Enfermera. Profesora Asociada en Ciencias de la Salud. Departamento de Enfermería y Fisioterapia. \\ Universidad de Alcalá \\ JUAN LUIS Miguel Díaz \\ Sociológo \\ SONSOLES RODRÍGUEZ DE LA PARRA \\ Enfermera. Profesora Titular. Departamento de Enfermería y Fisioterapia. Universidad de Alcalá
}

Correspondencia: Helena Hernández Martínez

$\mathrm{C} /$ Braulio Vivas $4,5^{\circ} \mathrm{D}$

28806 Alcalá de Henares

Madrid

\section{RESUMEN}

El tránsito entre una sociedad de pensamiento netamente mágico a una de carácter racional se caracteriza porque la humanidad en su acepción más amplia, se fundamenta en la ciencia y se nutre de ella. Como todo proceso que implica una relación interpersonal, la relación de la enfermería con los demandantes de sus cuidados es una interacción recíproca y cargada de factores sociológicos.

En este trabajo planteamos la noción de salud mental desde la perspectiva de "lo social", en relación directa con los entornos culturales cambiantes y multivariados, cuyas funciones y estructuras constituyen cada sistema cultural. Un recorrido por la evolución del pensamiento filosófico y social de la humanidad, del concepto de salud mental, y del cuidado enfermero, nos muestra las relaciones existentes entre ambos. La percepción de enfermedad mental ha ido cambiando de acuerdo con la evolución del pensamiento social, y la profesión enfermera, integrante de esa sociedad, ha ido desarrollando su pensamiento y su paradigma como disciplina.

Palabras clave: pensamiento social, salud mental, enfermedad mental, enfermería, evolución.

\section{DIFFERENCE BETWEEN A SOCIETY OF PURE MAGIC THOUGHT AND A RATIONALISTIC THOUGHT}

\section{SUMMARY}

The difference between a society of pure magic thought and that of a rationalistic character is characterized by its humanity fundamentally based in science and sustained by science. As in any process implying an interpersonal relationship, the relation between nursing and the demanding users of health care, is of a reciprocal nature and as a consequence, full of sociological factors. In this work, we present the notion of mental health from a "social" perspective closely linked to those multi-cultural backgrounds whose different structures and functions contribute to the overall cultural system.

A survey performed of mankind's philosophical and social thought regarding the concept of mental health and nursing care shows us their inter-relationship. The perception of mental illness has developed according to the evolution of social thought; and the nursing profession, part of this society, has also developed its thought and paradigm as a new discipline.

Key words: social thought, mental health, mental illness, nursing evolution. 


\section{INTRODUCCIÓN}

La percepción de la enfermedad mental ha ido cambiando a través del tiempo en concordancia con la evolución del pensamiento social.

El pensamiento humanista del siglo XVII colo$\mathrm{ca}$ al ser humano en el centro del universo. Se asiste al inicio de un nuevo concepto de persona, como unidad integrada por un conjunto de nexos entre lo somático, lo psicológico y lo social.

Bajo la influencia de esta concepción del ser humano, la enfermedad mental comienza a percibirse como una alteración dentro del continuo salud-enfermedad, que se analiza desde diversas disciplinas: psicología, psiquiatría, antropología y sociología, quedando relegado el valor mágico y demoniaco que se le había atribuido históricamente.

De acuerdo con este planteamiento, para establecer una relación de ayuda terapéutica, las enfermeras han de desvelar el perfil psicosocial de las personas y familias a las que atienden, sus procesos y estructuras mentales, la influencia que los factores condicionantes ejercen sobre su estado de salud, y el rol que dichas personas y sus familias deben desempeñar en el entorno en que se desarrollan.

\section{PERSPECTIVA SOCIAL DE LA SALUD MENTAL}

El proceso psicosocial es muy importante en el desarrollo de los trastornos de salud mental, porque la percepción y atribución de los hechos, se traslada a la esfera de lo social.

Para poder percibir y adaptarse a la realidad cambiante, el ser humano necesita de sus redes familiares y sociales. Por el contrario, el aislamiento, la reclusión o la marginación favorecen la inadaptación.

En la sociología y la psicología, la normalidad se asume como una constante que no se desvía de un valor, el cuál no está definido, pues la normalidad depende del modelo de ecosistema social concreto. Entre las "personas normales" existen variaciones de comportamiento y salvo en grandes desviaciones de la norma, que prevalece en cada cultu- ra como valor, la frontera entre lo normal y lo anormal es difusa. Son las exigencias específicas de cada tiempo y lugar el único referente que nos sirve para delimitar esta frontera.

La salud no es un concepto estático en la estructura social, depende de las posibilidades de adaptación al medio físico y social, un equilibrio relacional positivo. Por todo ello, el estado de salud de cada persona estará en la esfera de lo relativo, de la percepción de lo individual y del grupo social.

Al hablar de salud mental, hablamos de tolerancia, integración, reinserción... Esta relación entre el ser humano, su ambiente, su entorno, es materia de reflexión porque el "ser social" que cada uno de nosotros somos, depende de la organización en la que se encuentra, de la tolerancia de ésta y de la capacidad de adaptación de la persona a ella.

De esta manera, nos acercamos al estudio antropológico de la enfermedad mental, enfoque científico que considera las mismas enfermedades en culturas distintas; el resultado de sus estudios describe como varían las manifestaciones de los trastornos mentales en función del proceso endocultural del grupo.

Para el funcionamiento social de la persona es prioritaria la salud' y especialmente la salud mental, evidenciándose en la cantidad y calidad de las relaciones y apoyos sociales de la persona. La salud mental se ve afectada por el estrés relacional existente en los acontecimientos cotidianos vitales. Los estudios científicos resaltan la importancia de las relaciones con los demás y el apoyo familiar y social como claves para reducir o evitar los efectos perniciosos de los factores exógenos estresantes.

\section{EVOLUCIÓN DEL PENSAMIENTO SOCIAL Y DEL CONCEPTO DE SALUD MENTAL}

En las sociedades tradicionales primitivas el individuo "anormal" era tolerado por el grupo siempre que participara en los ritos culturales y se integrara en las prácticas productivas. Sus disfunciones eran asumidas, incluso como algo mágico y no había necesidad de actuar sobre él. 
Con el desarrollo de las religiones, entre ellas el cristianismo, las enfermedades son interpretadas como manifestaciones de castigos divinos, y las alteraciones de la salud mental se identifican con posesiones demoniacas, lo que conduce a la marginación, separación del grupo e incluso el sacrificio de las personas con trastornos mentales.

Tras el triunfo de las ideas de la Ilustración y la Revolución Francesa, se sustituye el poder religioso por el poder civil. Los trastornos mentales comienzan a observarse como una enfermedad más que precisa ser tratada por los profesionales de la salud.

El romanticismo y el posterior desarrollo de las grandes teorías sociológicas -el idealismo de Hegel, el marxismo, el positivismo de Comte, el utilitarismo de Max Weber- postulan una nueva visión del ser humano y la influencia que ejerce en el desarrollo del mismo su interrelación con el entorno, la cultura, el aprendizaje y los valores y normas prevalentes en el grupo social.

La sociedad industrial con su sistema normativo y formativo, favorece el fracaso social de sus miembros, para más tarde excluirlos e ignorarlos por resultar incómodos, en lugar de plantearse su reintegración en la sociedad.

La Escuela de Chicago y los trabajos de Talcott Parson $^{2}$ estudian las conductas desviadas, el rol del enfermo mental, la influencia de las instituciones psiquiátricas y la posible equiparación de la enfermedad psíquica a un trastorno de adaptación, utilizando los términos "enfermo psíquico" y "ser inadaptado".

Entre los años 1950-1975, el número de trastornos mentales medicalizados aumentó en un 40\%. El ritmo impuesto por la organización social actual con sus demandas y problemas político-económi$\cos$ y sociales favorece la presencia de trastornos mentales en las sociedades occidentales. Un $25 \%$ de los parados sufren algún tipo de alteración mental, un $7 \%$ de los niños sufren problemas emocionales y en los grupos marginados y excluidos del sistema social -inmigrantes, pobres, y adictos a drogas- son cada vez más frecuentes los trastornos de la personalidad.

Los trabajos de Durkheim postulan que este aumento de los trastornos mentales es debido a la "anomia" que experimentan las personas bajo la influencia del individualismo propugnado por la sociedad postindustrial, ya que el aislamiento del individuo es contrario a la necesidad de pertenencia al grupo, requisito indispensable para el desarrollo del ser humano, que sí podía satisfacerse en la sociedad preindustrial. En sus trabajos se estudia la dificultad que supone pasar del sentido de pertenencia al grupo característico de la sociedad preindustrial, al individualismo característico de la sociedad postindustrial.

La concepción marxista describe como los procesos productivos de la era industrial traen consigo la "alienación" del ser humano y por tanto el aumento de desadaptación social y de enfermedades mentales.

\section{LA ATENCIÓN AL ENFERMO MENTAL}

De acuerdo con la evolución del pensamiento social, la atención al enfermo mental ha pasado por las siguientes etapas:

- Etapa de marginalidad, aislamiento y agresión. No hay ningún tipo de atención y/o cuidados al enfermo mental.

- Etapa asilar: bajo la influencia de las ideas humanistas del siglo XVIII, los "locos" han de ser protegidos, se les ingresa de por vida en instituciones, antiguas leproserías, para cubrir sus necesidades básicas. De este modo la sociedad se olvida de ellos al ocultarlos y aislarlos.

- Etapa institucional: a partir de 1800, con la evolución del pensamiento social, antes mencionada, los trastornos mentales se consideran una enfermedad. Los médicos, inexistentes hasta entonces en instituciones psiquiátricas, van asumiendo el liderazgo del área. Se crean nuevas instituciones para rehabilitar al enfermo, en las que priman la disci-

2.- Citado por: Hewstone M. y col. Introducción a la psicología social. Ariel. Barcelona 1990.

3.- Citado por: Harris M. Introducción a la antropología general. Alianza Editorial. Madrid 1991. 
plina y el orden interno. Es la etapa de desarrollo de los manicomios.

- Etapa social: en 1933 los sociólogos de la Escuela de Chicago comienzan a estudiar al enfermo mental en su entorno social. Se analiza la microsociedad y cultura de los centros psiquiátricos, el deterioro y la cronificación de los enfermos ingresados, llegando a plantear la "neurosis institucional". Los hospitales mentales continúan siendo centros de larga estancia en los que se recluye al enfermo y se le aleja de su entorno habitual. Se inicia la psicoterapia institucional.

Tras la Segunda Guerra Mundial, el interés de las disciplinas que estudian al ser humano se centra en el medio ambiente, el entorno, la clase social, las redes familiares, la organización, las estructuras sociales, etc.

En 1959, la OMS recomienda las funciones preventivas y terapéuticas que debe asumir la comunidad, considerando la atención a la salud mental un problema de ámbito social.

- Etapa comunitaria: la Ley General de Sanidad de 25 de Abril de 1986 integra la asistencia psiquiátrica en el Sistema Nacional de Salud, iniciándose la asistencia de los enfermos mentales en los hospitales generales, centros de salud mental, hospitales de día, unidades de agudos, etc.

El comité de expertos en salud mental de la OMS, basándose en la Teoría General de Sistemas, define la salud mental en los siguientes términos: "no basta con carecer de defectos mentales, tampoco con ser capaz de adaptarse al medio, sino que implica la capacidad para conseguir unas relaciones armoniosas con los otros y para participar o contribuir de manera constructiva a la modificación del medio social o físico"

Este cambio se debe al reconocimiento del papel fundamental de la familia como responsable de los cuidados, y de la necesidad de la rehabilitación y reinserción del enfermo mental en su entorno habitual, la no marginalidad y un Sistema Nacional de Salud universal y equitativo.

\section{SALUD MENTAL Y CUIDADOS ENFERMEROS}

En las sociedades primitivas el objetivo del cuidado era promover la vida y proteger al individuo para así garantizar la supervivencia del grupo. El cuidado recaía en la mujer que había acumulado experiencias en la atención a los miembros del grupo en las diferentes etapas del ciclo vital: nacimiento, crianza, madurez y muerte. El cuidado era asumido en el seno familiar y grupal.

Como consecuencia de los valores desarrollados por el cristianismo -caridad, amor al prójimo, entrega, etc.- algunas personas convierten su vida en un acto de amor a Dios, a través del cuidado de sus semejantes. Los cuidados ya no son proporcionados por los miembros del grupo, sino por personas ajenas al mismo. Surgen las órdenes religiosas dedicadas al cuidado de los enfermos, incluidos los enfermos mentales.

Las ideas de la Ilustración priorizan el papel de la razón, y ponen en duda el saber de la tradición y de la autoridad en todas las ciencias. Pero los cuidadores de la época no se plantean el porqué del ser y del quehacer enfermero.

En la etapa asilar e institucional, el cuidado de los enfermos mentales estaba en manos de órdenes religiosas, cuyo trabajo principal consistía en mantener el orden, la organización y la limpieza del asilo, además de ocuparse de la custodia de los internos.

En el siglo XIX Max Weber, entre otros autores, señala que la finalidad de la ciencia es la búsqueda de la verdad, la descripción y la explicación de los hechos; explicaciones que la enfermería no se planteará hasta mediados del siglo $\mathrm{XX}$, que es cuando aparecen las primeras teorías sobre la disciplina enfermera. Sin embargo, no sería justo olvidar la figura de Florence Nightingale, que ya en el siglo XIX había iniciado en solitario la definición y los fundamentos de la enfermería como profesión.

A partir de 1950, la disciplina enfermera comienza a desarrollarse como ciencia, estudiando los fenómenos y estableciendo las relaciones entre los elementos que componen las teorías. Los modelos enfermeros facilitan la aplicación de las teorías ya conformadas, y constituyen el marco de referencia que proporciona las directrices para la práctica enfermera. 
La influencia de los valores predominantes en la sociedad y del momento histórico y científico en que se han desarrollado las teorías enfermeras, han dado lugar a diferentes Escuelas de Pensamiento Enfermero.

A principios de los años 60, el papel de las enfermeras en los "manicomios", -practicantes y ATS en su mayoría masculinos- consistía principalmente en la custodia de los internos y la ayuda al psiquiatra en la administración de los tratamientos electroconvulsionantes, curas de Sakel y aislamientos.

A mediados de los años 80 se inicia un cambio en la concepción del cuidado enfermero al paciente psiquiátrico, acorde con el desarrollo de la disciplina enfermera y con la evolución del concepto de enfermedad a salud mental. El objetivo de los cuidados enfermeros consiste ahora en ayudar a la persona con problemas de salud mental potenciando sus recursos personales y familiares para desarrollar su capacidad para el autocuidado, su adaptación y su reinserción social. La enfermera interactúa con la persona y su familia para cubrir tanto sus necesidades básicas de salud, como las específicas de su alteración, a través de una relación de ayuda terapéutica en la que el foco de atención es la persona con problemas de salud.

\section{CONCLUSIONES}

- El enfoque sociológico nos acerca a la comprensión del enfermo mental desde un campo crítico, desde la discusión de las fronteras de la normalidad. La OMS en 1959 apela a ese límite de normalidad mental, en función de la reacción del colectivo social ante un determinado trastorno.

- La atención al enfermo mental ha ido transformándose de acuerdo con las corrientes de pensamiento y los valores de la sociedad prevalentes en cada momento.

- La enfermería inicia su desarrollo como disciplina a partir de 1950, y también se ha visto influi- da por las corrientes de pensamiento en cada momento de su evolución.

- Actualmente el objetivo del cuidado enfermero, debe dirigirse a potenciar los recursos personales y comunitarios, ayudando a los pacientes y sus familias a desarrollar habilidades que promuevan su autonomía, su autocuidado y su inserción paulatina en la vida social.

\section{BIBLIOGRAFÍA}

Fmelser NJ. (1990) Teoría sociológica: Análisis histórico y formal. Espasa Calpe. Madrid.

Harris M. (1981) Introducción a la antropología general. Alianza Editorial. Madrid.

Hernández Conesa J. (1995) Historia de la Enfermería. MacGraw-Hil Interamericana. Madrid

Hewstone M. y col. Introducción a la psicología social. Ariel. Barcelona 1990.

Kérouac, S., Pepin, J., Ducharme, F., Duquette, A., Major, F. (1996) El pensamiento enfermero. Masson. Barcelona.

Marriner-Tomey, A. (1994) Modelos y teorías en enfermería. Mosby/Doyma. Madrid

OMS. (1959) Psiquiatría social y actitudes de la colectividad. Serie Informes Técnicos $n^{\circ} 177$. Ginebra

OMS. (1959) Higiene Mental. Serie Informes Técnicos $n^{\circ}$ 9. Ginebra

OMS. (1993) Descripciones clínicas y pautas para el diagnóstico. Trastornos mentales y del comportamiento.

Clasificación

Internacional de las Enfermedades Mentales. Ginebra

San Martín H.(1979) Ecología humana y salud. Copilco. México

VVAA. (1997) Psiquiatría social y comunitaria. ICEPPS. Las Palmas

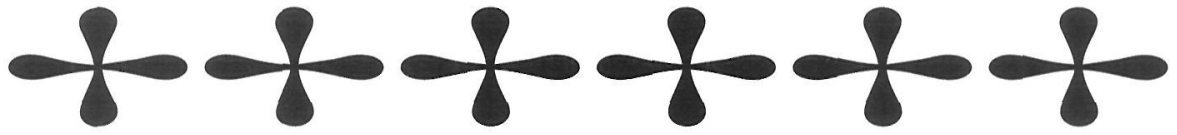

